\title{
SmartHearing Aid: Um simulador de aparelho auditivo para smartphone
}

\author{
André Ludwig Machado de Almeida ${ }^{1}$ \\ Lucas Toshio de Souza Miki² \\ Luiz Antônio Baggiotto Rubin ${ }^{3}$ \\ Samuel Ariel Müller ${ }^{4}$ \\ Caroline Inês Lisevski ${ }^{5}$ \\ Tiago Baptista Noronha ${ }^{6}$
}

\section{RESUMO}

Os espaços urbanos contemporâneos possuem cada vez mais ruídos, produzindo danos à audição. Com os avanços da tecnologia surgiram métodos capazes de amenizar o problema da perda auditiva, como o uso de aparelhos auditivos. Este trabalho visa o desenvolvimento de um aplicativo gratuito para smartphone na plataforma Android, que permite utilizar um fone de ouvido como aparelho auditivo.

Palavras-chave: deficiência auditiva, aplicativo, fone de ouvido.

\section{INTRODUÇÃO}

A audição é considerada um dos principais sentidos presente na vida humana. É através dela que uma criança desenvolve a fala, a capacidade de ler, escrever e também suas habilidades sociais.

A perda auditiva vem se tornando incapacitante para muitas pessoas. Dados de 2019 da World Health Organization (WHO) afirmam que cerca de 466 milhões de pessoas no mundo têm deficiência auditiva incapacitante e uma projeção para 2050, é de que 900 milhões de pessoas sejam afetadas por algum tipo de perda auditiva [1].

Um dos fatores etiológicos destas perdas auditivas é o processo de envelhecimento humano, o qual inicia por volta dos 50 anos de idade. Cerca de $23 \%$ da população entre 65 e 75 anos apresentam perda auditiva relacionada à idade, e a partir dos 75 anos, esta porcentagem aumenta para 40 \% [2]. A diminuição auditiva relacionada ao envelhecimento é denominada de presbiacusia. Ela pode ser classificada em quatro tipos: (i) Presbiacusia sensorial, (ii) Presbiacusia neural, (iii) Presbiacusia metabólica e (iv) Presbiacusia mecânica [3]. A presbiacusia sensorial é a mais comum e é referente à perda auditiva neurossensorial bilateral e simétrica, determinando perda auditiva em sons agudos. A presbiacusia neural é

\footnotetext{
${ }^{1}$ IFSul - Instituto Federal Sul-rio-grandense - Câmpus Charqueadas - Brasil.

2 IFSul - Instituto Federal Sul-rio-grandense - Câmpus Charqueadas - Brasil.

3 IFSul - Instituto Federal Sul-rio-grandense - Câmpus Charqueadas - Brasil.

${ }^{44}$ IFSul - Instituto Federal Sul-rio-grandense - Câmpus Charqueadas - Brasil.

${ }^{5}$ IFSul - Instituto Federal Sul-rio-grandense - Câmpus Charqueadas - Brasil.

${ }^{6}$ IFSul - Instituto Federal Sul-rio-grandense - Câmpus Charqueadas - Brasil.
} 
caracterizada pela perda de neurônios cocleares, que pode ser relacionada com dificuldade de coordenação motora e déficits cognitivos. A presbiacusia metabólica é associada à atrofia das estrias vasculares enquanto que a presbiacusia mecânica ocorre devido ao enrijecimento da membrana basilar e alteração nas características de ressonância do ducto coclear.

O declínio da acuidade auditiva pode ser influenciado e agravado por doenças sistêmicas, alcoolismo, exposição ao ruído, estresse, ototoxicidade, distúrbios metabólicos e vasculares, e fatores hereditários [4].

Segundo World Health Organization (WHO), a perda auditiva impacta diariamente na vida das pessoas, causando sentimentos de solidão, isolamento, frustração, deterioramento cognitivo e comunicativo. O primeiro tratamento a ser indicado é a utilização de aparelhos auditivos individuais $[1,5]$.

O custo-benefício dos aparelhos auditivos está ligado diretamente ao poder de processamento, o qual está relacionado com a quantidade de canais de compreensão que garantem melhor gerenciamento da audibilidade específica por frequências. Além disso fatores como ergonomia, tecnologias implementadas para diminuição de ruídos e interferências, recursos direcionados aos usuários e grau de deterioração nos ouvidos influenciam no valor final do equipamento.

O valor dos aparelhos auditivos com um bom desempenho possui valores entre $\mathrm{R} \$ 2.500 \mathrm{a}$ R\$ 18.000 [6] e, para uma grande parcela da população, é inviável a sua aquisição.

Segundo a Revista Época, no Brasil a quantidade de smartphones ultrapassam os 230 milhões de dispositivos [7]. Considerando esses dados, o objetivo desse projeto foi 0 desenvolvimento de um software para smartphone capaz de tornar um fone de ouvido em um aparelho auditivo. Dessa maneira toda população com algum tipo de perda auditiva e em qualquer condição socioeconômica possui acesso ao recurso, o qual pode melhorar a qualidade de vida da pessoa. Uma das vantagens do trabalho é a possibilidade do usuário escolher qual fone de ouvido the oferece a melhor experiência e conforto.

\section{METODOLOGIA}

O áudio é definido como um sinal temporal contínuo no tempo de amplitude analógica [8]. Esses sinais podem ser estudados e manipulados através de ferramentas matemáticas como Transformada de Laplace, Série de Fourier, Transformada de Fourier e suas Transformadas Inversas. Algumas das manipulações feitas são multiplicação ou convolução do sinal para obter resultados de frequência, amplitude, inversão da função, compressão e expansão da onda em relação ao seu período. A partir disso é possível aplicar filtros para manipular uma onda sonora. 
Figura 1: Gráfico da frequência do sinal sonoro versus Amplitude. [9]

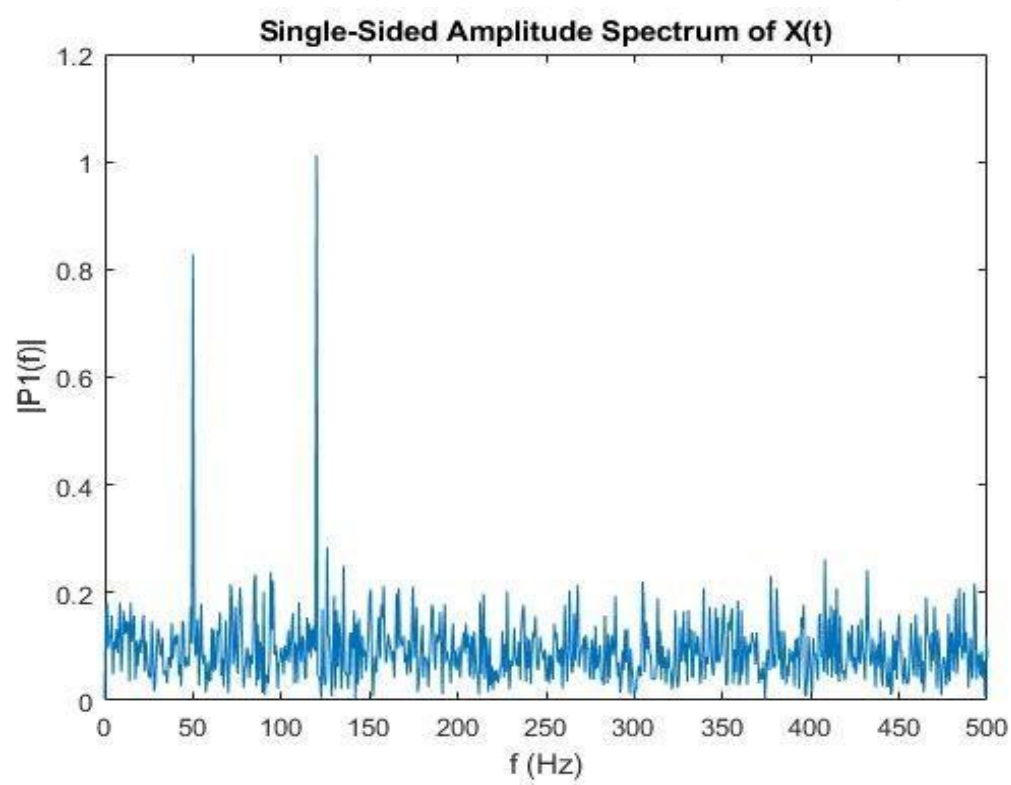

Na Figura 1 é apresentado um gráfico da frequência do sinal sonoro versus a amplitude da onda. Como o estudo está relacionado à um sistema sonoro real, considera-se que a amplitude seja a intensidade de estímulos que o órgão recebe para a percepção de sons. A intensidade sonora é medida em decibéis $(\mathrm{dB})$ e, para uma pessoa que possui uma audição normal, o limiar de audição varia entre 0 e 20 dB.

Figura 2: Script de captura de áudio.

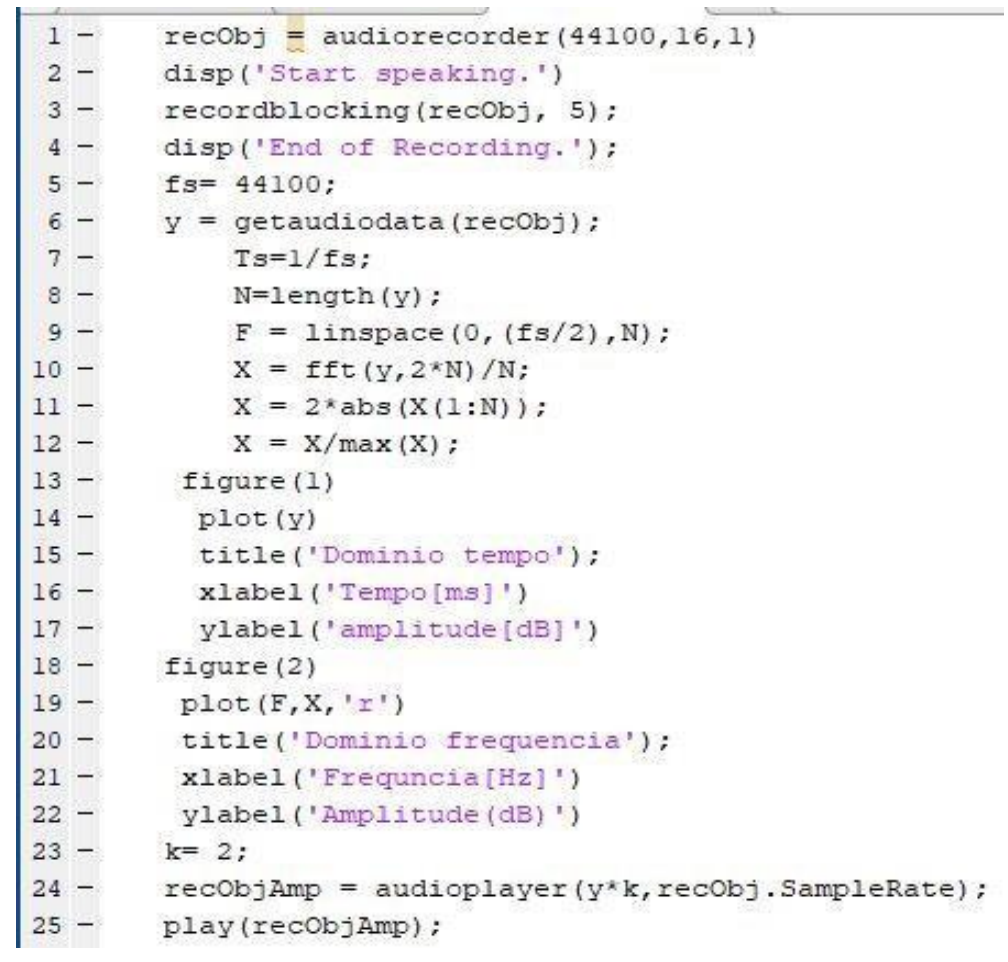

Para captar e manipular o sinal sonoro através da aplicação de filtros, foi usado o software de cálculo numérico Matlab. A partir disso, foi programado um script (Figura 2), no qual o microfone de um computador gravou um áudio de cinco segundos com a frase "Bom dia", repetida 3 vezes, e dois ruídos contínuos, reproduzido pelo aplicativo para smartphone "Gerador de frequências" na faixa de 1000 Hz e 10000 Hz. O áudio foi guardado em um 
vetor, pois a função é reproduzida graficamente em n-amostras com a uma taxa de amostragem de 44100 amostras por segundo, com amplitude de potência máxima aproximada a 1. Para fins demonstrativos, não foi usado valores em $\mathrm{dB}$ na ordem logarítmica. Foram usadas as funções audiorecorder juntamente com recordblocking, que capturam o áudio através de um microfone e estipulam o tempo de captura. A função getaudiodata foi aplicada para retornar dados do áudio gravado e associados ao audiorecorder para o objeto recObj em um vetor double, possibilitando a normalização para aplicação da FFT, assim atribuído respectivamente a abscissa ' $y$ '.

\section{RESULTADOS E DISCUSSÕES}

Utilizando o script de gravação de áudio (Figura 2) para salvar as informações do sinal captado, percebe-se que ele está disposto no domínio do tempo. Isso faz com que essas informações apresentem vetores de números complexos. A partir dessas informações foi possível extrair os ângulos de fase da onda, que, se não levados em consideração ao aplicar algum tipo filtro, acarretarão em distorções no áudio tratado.

No sinal sonoro coletado pelo software (Figura 3), não é possível determinar uma função característica do sinal, inviabilizando o uso da Transformada de Laplace e de Séries de Fourier para análise. Como o sinal sonoro possui frequências indefinidas, a Transformada de Fourier é o método mais adequado de análise.

Figura 3: Gráfico áudio captado pelo script de gravação, amostras versus amplitude

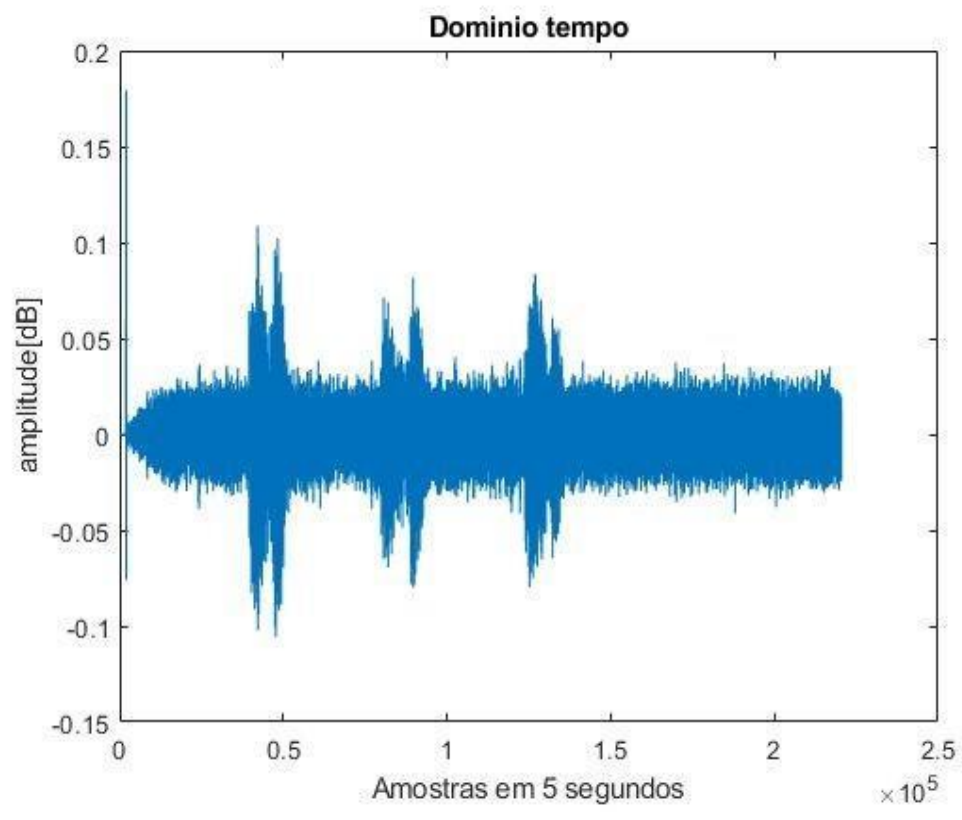

O Matlab foi utilizado para analisar o comportamento do sinal captado. Como a entrada é em função do tempo-amplitude, não foram obtidos bons resultados. Assim, foi realizada uma normalização do sinal de entrada e aplicada a Fast Fourier Transform (FFT) (Figura 4), que é um algoritmo mais eficiente para calcular a Discrete Fourier Transform (DFT), tornando a FFT uma melhor opção para o processamento digital de sinais, assim convertendo um sinal em seu domínio original no tempo para o domínio da frequência. 
Figura 4: Gráfico Fast Fourier Transform (FFT), domínio Frequência versus amplitude.

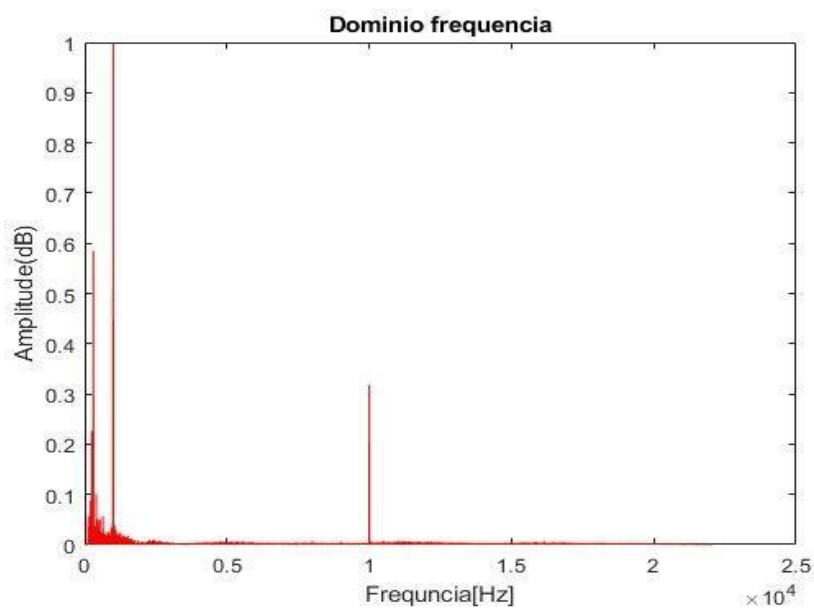

Dentre os filtros, a multiplicação é o melhor método para avaliar a escala em decibéis na frequência. Assim, multiplicando o áudio reproduzido remotamente por uma constante, amplificou-se a abscissa da amplitude, podendo então alterar a constante para que a escala em decibéis seja apresentada.

Buscando aproximar cada vez mais de um aparelho auditivo simples, multiplicou-se o vetor " $y$ " por duas vezes seu valor nominal, e o áudio foi reproduzido (Figura 5). Porém, como captava o som integralmente, tanto a voz quanto os ruídos eram amplificados, além das interferências do hardware. Sem tirar esses ruídos, a utilização desse aparelho auditivo seria incômoda quando utilizado por longos períodos de tempo.

Figura 5: Áudio amplificado, número de amostras versus amplitude.

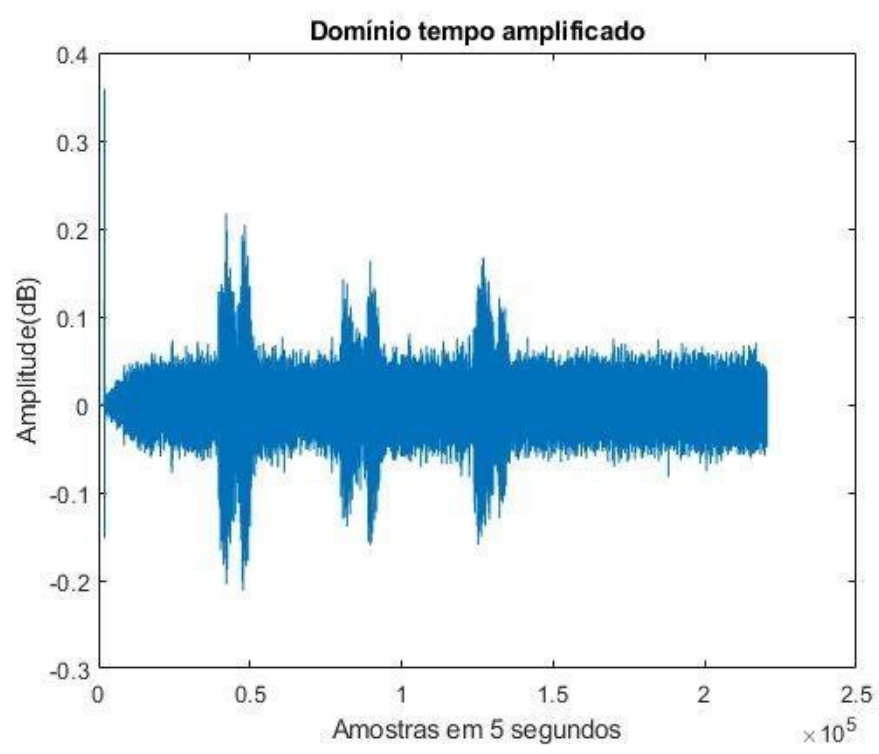

O script mostrado na Figura 1 não se equipara a um aparelho auditivo pelo fato de captar 0 áudio durante 5 segundos e, somente após ser construído o vetor " $y$ " (Figura 6), dá-se o início à reprodução do áudio. Esse vetor "y" tem dimensão correspondente à taxa de amostragem multiplicado pelo tempo de gravação. No caso trabalhado, a taxa de amostragem foi de 44100 amostras durante 5 segundos, totalizando 220500 amostras. 
Figura 6: Vetor de amostras do script da Figura 1.

\begin{tabular}{|c|c|c|c|}
\hline \multicolumn{3}{|c|}{2 Editor - Untitled.m } & \\
\hline re & $x \longdiv { y }$ & $x$ & \\
\hline \multicolumn{4}{|c|}{$\boxplus 220500 \times 1$ double } \\
\hline & 1 & & 2 \\
\hline 220485 & -0.0043 & & \\
\hline 220486 & -0.0146 & & \\
\hline 220487 & -0.0144 & & \\
\hline 220488 & -0.0152 & & \\
\hline 220489 & -0.0123 & & \\
\hline 220490 & -0.0152 & & \\
\hline 220491 & -0.0212 & & \\
\hline 220492 & -0.0244 & & \\
\hline 220493 & -0.0201 & & \\
\hline 220494 & -0.0131 & & \\
\hline 220495 & -0.0184 & & \\
\hline 220496 & -0.0194 & & \\
\hline 220497 & -0.0199 & & \\
\hline 220498 & -0.0152 & & \\
\hline 220499 & -0.0121 & & \\
\hline 220500 & -0.0147 & & \\
\hline
\end{tabular}

Logo, a possibilidade de aplicar os filtros no domínio do tempo deixa a desejar na velocidade de processamento, pois é necessário que haja uma convolução com a aplicação das integrais mais complexas.

Para contornar tal problema, foi implementado um novo script (Figura 7) e com o auxílio da biblioteca de áudio do Matlab e um laço de repetição while, no qual foi definido o tempo de reprodução. Com isso foi possível capturar e reproduzir o áudio amplificado em tempo real.

Figura 7: Captura de áudio e reprodução em tempo real.

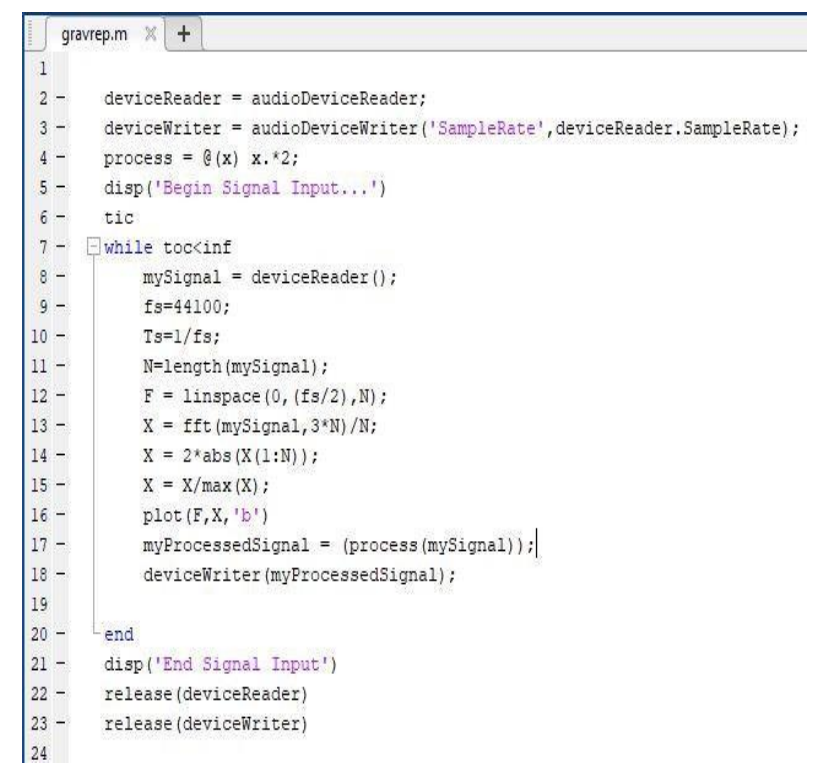

O algoritmo foi eficaz quando inicializado em um Macbook com processador intel core i5, porém demonstrou um delay considerável quando inicializado em um notebook Windows dual core. Para que o simulador funcione de forma eficiente, é necessário que o delay seja mínimo, de modo que o usuário não se sinta desconfortável. Delays de centésimos de segundos levam à insatisfação do deficiente auditivo. 


\section{CONCLUSÃO}

O aparelho auditivo simulado comporta filtros em bandas de áudio com transmissão embutida. Mas, devido ao espaço limitado, apresenta atrasos e baixa qualidade na sua transmissão. $\mathrm{O}$ aplicativo final objetiva uma eficiência maior que os dispositivos de mercado e menor custo, visando a inclusão social.

A qualidade final do produto está relacionada à utilização do melhor método de filtragem, sua eficiência e sem privar o usuário de usufruir do som ambiente, a não ser que ele opte pelo mesmo através de uma implementação de ambientação.

A necessidade de estudos mais aprofundados acerca da aplicabilidade dos filtros se dará na continuidade do projeto.

\section{REFERÊNCIAS}

[1] Deafness and hearing loss. World Health Organization, 2019. Disponível em: <https://www.who.int/news-room/fact-sheets/detail/deafness-and-hearing-loss> Acesso em: 23 de agosto de 2019.

[2] Rev CEFAC, v.11, Supl1, 106-115, 2009 <https://forl.org.br/Content/pdf/seminarios/semin ario_52.pdf> Acessado em 23 de agosto de 2019.

[3] Barros PFS, Queiroga BAM. As dificuldades encontradas no processo de adaptação de aparelhos de amplificação sonora individual em indivíduos idosos. Rev CEFAC. 2006; 8(3):375-85.

[4] Almeida K, Iório MCM. Próteses auditivas: fundamentos \& aplicações clínicas. São Paulo: Lovise; 1996.

[5] Moreira, Paula P. Preço de aparelho auditivo em 2018: resultado da pesquisa entre os leitores do Crônicas da Surdez, 2018. Disponível em: <https://cronicasdasurdez.com/precoaparelho-auditivo-2018-1/> Acessoem: 23 de agosto de 2019.

[6] Brasil tem 230 milhões de smartphones em uso. Época Negócios, 2019. Disponível em: <https://epocanegocios.globo.com/Tecnologia/noticia/2019/04/brasil-tem-230-milhoesde-smartphon es-em-uso.html> Acesso em: 23 de agosto de 2019.

[7] Lathi, B. P. Sinais e Sistemas Lineares. 2a edição. Bookman: São Paulo, 2006.

[8] MathWorks. Fast Fourier Transform. 2019. Disponível em: <https://www.mathworks.com/help/matlab/ref/fft.html> Acesso em: 23 de agosto de 2019. 